\title{
BMJ
}

\section{Perioperative epidural analgesia for major abdominal surgery for cancer and recurrence-free survival: randomised trial}

\author{
Paul S Myles, professor, ${ }^{1}$ director, ${ }^{2}$ Philip Peyton, consultant, ${ }^{3}$ Brendan Silbert, consultant, ${ }^{4}$ Jennifer Hunt, \\ research coordinator, ${ }^{1}$ John R A Rigg, retired consultant, ${ }^{5}$ Daniel I Sessler, professor and chair ${ }^{6}$ for the ANZCA \\ Trials Group Investigators
}

Department of Anaesthesia and Perioperative Medicine, Alfred Hospital, Melbourne, Australia

${ }^{2}$ Academic Board of Anaesthesia and Perioperative Medicine,

Monash University, Melbourne

${ }^{3}$ Department of Anaesthesia,

Austin Hospital, Heidelberg,

Australia

${ }^{4}$ Department of Anaesthesia, St Vincent's Hospital, Fitzroy,

Australia

${ }^{5}$ Australian and New Zealand College of Anaesthetists Trials Group, Melbourne

${ }^{6}$ Department of Outcomes Research, Cleveland Clinic,

Cleveland, USA

Correspondence to: P S Myles

p.myles@alfred.org.au

Cite this as: $B M J$ 2011;342:d1491 doi:10.1136/bmj.d1491

\section{ABSTRACT}

Objective To compare long term recurrence of cancer and survival of patients having major abdominal surgery for cancer.

Design Long term follow-up of prospective randomised controlled clinical trial in which patients were randomly assigned to receive general anaesthesia with or without epidural block for at least three postoperative days.

Setting 23 hospitals in Australia, New Zealand, and Asia. Participants 503 adult patients who had potentially curative surgery for cancer.

Main outcome measure Cancer-free survival (analysis was by intention to treat).

Results Long term follow-up data were available for $94 \%$ $(n=446)$ of eligible participants. The median time to recurrence of cancer or death was 2.8 ( $95 \%$ confidence interval 0.7 to 8.7$)$ years in the control group and $2.6(0.7$ to 8.7 ) years in the epidural group $(\mathrm{P}=0.61)$. Recurrencefree survival was similar in both epidural and control groups (hazard ratio 0.95, 95\% confidence interval 0.76 to $1.17 ; \mathrm{P}=0.61$ ).

Conclusion Use of epidural block in abdominal surgery for cancer is not associated with improved cancer-free survival.

Trial registration Australian New Zealand Clinical Trials Registry ACTRN12607000637448

\section{INTRODUCTION}

Even with the best technique, surgery for cancer is usually associated with release of cancer cells into the lymphatic system and bloodstream, and a large fraction of patients already have micrometastases and scattered cancer cells at the time of surgery. ${ }^{1}$ Whether residual postoperative cancer results in clinical metastases depends largely on the balance between antimetastatic immune activity and the tumour's ability to seed, proliferate, and attract new blood vessels. ${ }^{2}$ In practice, the immune system and other host defences often fail to neutralise minimal residual disease; consequently, local recurrence and metastatic disease remain common after surgery.
At least three perioperative factors threaten complete surgical eradication of cancer. The first is surgery itself, which releases cancer cells into the circulation, ${ }^{1}$ depresses cell mediated immunity including functions of cytotoxic $\mathrm{T}$ cells and natural killer cells, ${ }^{3}$ reduces circulating concentrations of tumour related antiangiogenic factors (such as angiostatin and endo statin), ${ }^{4}$ increases concentrations of pro-angiogenic factors such as vascular endothelial growth factor, ${ }^{5}$ and releases growth factors that promote local and distant growth of malignant tissue. ${ }^{2}$ The second factor is anaesthesia itself, which impairs many immune functions, including functions of neutrophils, macrophages, dendritic cells, T cells, and natural killer cells. ${ }^{6}$ The third factor is opioids, which are given to control surgical pain. Opioids inhibit both cellular and humoral immune function in humans. ${ }^{6}$ Furthermore, morphine is pro-angiogenic and promotes growth of breast tumours in rodents. ${ }^{7}$ Consequently, non-opioid analgesia helps to preserve the function of natural killer cells in animals and humans and reduces metastatic spread of cancer in rodents. ${ }^{8}$

Regional anaesthesia (intraoperative block of nociception) and analgesia (postoperative pain relief) attenuate or prevent each of these adverse effects. For example, regional anaesthesia largely prevents the neuroendocrine stress response to surgery by blocking afferent neural transmission from reaching the central nervous system and activating the stress response and by blocking descending efferent activation of the sympathetic nervous system, ${ }^{9}$ so little if any opioid analgesia is needed. Regional analgesia also reduces release of endogenous opioids. ${ }^{10}$ Consequently, little opioid induced immune impairment occurs. ${ }^{9}$ As might be expected, surgical stress is attenuated better by regional than by general anaesthesia. Consequently, natural killer cell function is better preserved and metastatic load to the lungs is reduced in a rat model of breast cancer metastasis. $^{3}$

Available data thus suggest that regional block with local anaesthetic helps to preserve effective defences against progression of tumour by attenuating the 
surgical stress response, by reducing or eliminating the need for volatile anaesthetics, and by sparing postoperative opioids. Animal studies are consistent with this theory, showing that regional block can reduce the metastatic burden in animals inoculated with breast adenocarcinoma cells. ${ }^{311}$ Only small observational studies are available in humans; two reported beneficial effects with regional anaesthesia in breast cancer and prostate cancer, ${ }^{12}{ }^{13}$ whereas three others did not in colon cancer, prostate cancer, and cervical cancer ${ }^{14-16}$; another reported equivocal results in prostate cancer. ${ }^{17}$

We previously did a multicentre randomised trial comparing regional block in patients having major abdominal surgery, many of whom were having potentially curative resection of cancer, mostly colon cancer. ${ }^{18}$ Patients in this MASTER trial had surgery 9-15 years ago, providing a unique opportunity to assess their cancer status and long term survival. Our aim was to identify whether epidural block reduces recurrence of cancer and improves survival. Specifically, we tested the hypothesis that epidural block for patients with comorbidity having major abdominal surgery and apparently complete resection of cancer reduces the risk of recurrence of cancer and death.

\section{METHODS}

\section{Study design and participants}

A detailed description of the design and 30 day outcomes of the MASTER trial has been previously published. ${ }^{18-20}$ MASTER was a multicentre randomised clinical trial designed to test the hypothesis that combined epidural and general anaesthesia reduces the frequency of a composite end point of mortality and major postoperative complications compared with general anaesthesia and opioid analgesia. ${ }^{19}$ It enrolled 915 patients having major abdominal surgery between July 1995 and May 2001. Patients were randomly assigned to receive intraoperative epidural anaesthesia and postoperative epidural analgesia (epidural group) or postoperative intravenous opioid based analgesia (no epidural group); all patients received general anaesthesia during surgery.

The protocol provided guidelines for premedication, intraoperative monitoring, site of the epidural (to be selected by the consultant anaesthetist to match the planned incision), use of intraoperative and postoperative epidural local anaesthetics to provide epidural block during and after surgery, induction and maintenance of general anaesthesia, replacement of blood and fluids, optimisation of core temperature and respiratory and cardiac function, criteria for tracheal extubation, and immediate postoperative medical management. With the exception of some pelvic operations, all epidural catheters were inserted in the thoracic region. Postoperative analgesia in the nonepidural group was mostly achieved with patient controlled, opioid based analgesia. In the epidural group, postoperative analgesia was managed with continuous infusions of bupivacaine or ropivacaine, supplemented with fentanyl or pethidine. Epidural analgesia was typically continued for three days after surgery.

For the clinical assessment of epidural block, we recorded minimum and maximum heart rates and systolic blood pressures during surgery in all patients as indicators of the clinical efficacy of intraoperative epidural block. After surgery, we measured intensity of pain at rest and after coughing in all patients by using a $10 \mathrm{~cm}$ visual analogue scale twice daily for the first three postoperative days as an indicator of effective pain control. Morphine remains the most widely used intraoperative and early postoperative opioid analgesic. Opioid usage was not recorded in the original MASTER trial database. We were none the less able to determine morphine use in the initial 72 postoperative hours from a single high recruiting site. We converted other opioids to morphine sulphate equivalents at ratios of 10:1 (pethidine) and 1:100 (fentanyl).

In the original study, among 440 patients randomised to the non-epidural group $19(4.3 \%)$ had epidural analgesia established preoperatively or within 72 hours of surgery. Among 447 patients randomised to epidural analgesia, $29(6.5 \%)$ did not receive an epidural, but in only 13 of these had it been unsuccessfully attempted (failure rate 4.6\%). Among the remaining patients, the average duration for which the epidural remained in situ postoperatively was 74 hours. In this analysis, we analysed the height of epidural block achieved in the first three postoperative days and report maximal height as another indicator of effective postoperative epidural block. Use of nitrous oxide was not recorded in the original study, but the usual practice at that time was to include it in most cases.

An additional inclusion criterion for the follow-up study was that patients had complete surgical excision of cancer. Types of surgery included oesophagectomy, gastrectomy, hepatectomy, pancreatectomy, colectomy, nephrectomy, cystectomy, radical hysterectomy, and open prostatectomy. We excluded patients who were not having surgery for cancer (for example, aortic surgery) or in whom excision of the cancer was macroscopically incomplete, including those with distant metastasis at the time of surgery.

In this follow-up study, we collected additional data relating to type and size of cancer and extent of spread at the time of the original surgery. This required retrospective review of the medical records of all patients relating to the time of their original surgery, including pathology reports, formal reporting to cancer registries, operation reports, and discharge summaries. We used organ specific tumour-nodes-metastases staging and the American Joint Commission on Cancer tumour grading system to characterise the extent of malignancy and spread of cancer. ${ }^{21}$

\section{Follow-up of patients}

Additional ethics approvals enabled us to search existing databases for evidence of recurrence of cancer, death, and cause of death. We were sensitive to the possible distress associated with late follow-up of patients who had had surgery for cancer many years 
earlier, including to their families, and the patients' rights to confidentiality of hospital and cancer registry data. We therefore developed an algorithm that would minimise direct contact with patients or families and protect the confidentiality of individual patients' data. We sought follow-up data in the following order: the patient's medical record from the source hospital, hospital surgery or pathology databases (or both), the patient's general practitioner, state based cancer registry or national death index (or both), letter of introduction followed by telephone contact of the patient, and letter of introduction followed by telephone contact of the patient's next of kin. We used an approved script for the telephone contact with the patient or next of kin. The ethics committee from one centre amended the protocol to prevent patients being contacted by researchers, as some patients may not have been made aware of their original diagnosis of cancer; another centre permitted patients to be contacted only through the patient's general practitioner. All other centres' ethics committees approved the followup procedures as outlined in the protocol.

We also searched medical records and registries with a patient identification number, date of birth, and date of surgery. The Australian national death index records multiple causes of death. These include those conditions involved in the morbid train of events leading to death that were classified as the underlying cause, the immediate cause, or any intervening causes and those conditions that contributed to death but were not related to the disease or condition that caused death. We classified the cause of death as primarily due to cancer, cardiovascular disease, sepsis, and other causes.

\section{Study end points}

The primary end point of the study was cancer-free survival after surgery; the secondary endpoint was survival (all cause mortality). We calculated recurrence of cancer and survival from the date of surgery until the event occurred or until last known contact. We censored patients who were lost to follow-up at the time of last record. Research staff blinded to exposure status collected all outcome data.

A preliminary evaluation of the original MASTER trial database suggested that about 600 patients had complete surgical excision of a cancer and would be eligible for inclusion in this follow-up study. Most of these patients had primary colorectal cancer, for which five year survival is approximately 50\% and 12 year survival is less than $20 \%$. If epidural block reduced recurrence-free survival by $25 \%$, then 250 patients per group would provide $89 \%$ power with a two sided $\alpha$ of 0.05 . For a reduction from $50 \%$ to $37.5 \%$ (a relative reduction of $25 \%$ ) in cumulative five year survival, 247 patients per group (total 494) would be needed to provide $80 \%$ power with a two sided $\alpha$ of 0.05 . However, a relative reduction of a third (from $50 \%$ to $33 \%$ ) would require only 131 patients per group.

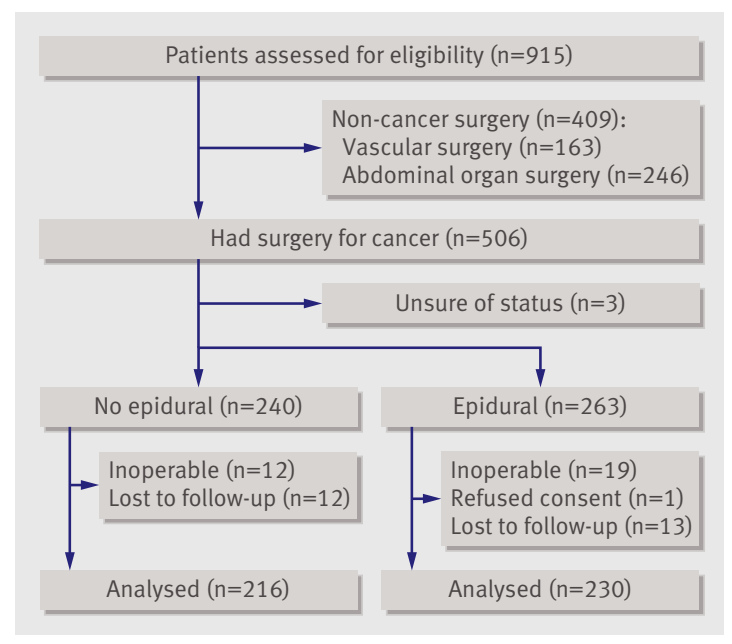

Fig 1| Trial profile

\section{Statistical analysis}

We based analyses on the intention to treat principle; we thus included all randomised patients who had potentially curative surgery for cancer. We constructed time to recurrence and, separately, time to death from any cause for both groups with KaplanMeier survival estimates and compared them by using the log-rank test. We used a Cox proportional hazards model to adjust for imbalances of potentially important covariates, with assessment of the requisite proportionality assumptions. We planned to include patients' sex, age, and type of surgery in our adjusted analyses and tests of subgroup interaction. Also, in view of recent publications suggesting a possible effect modification of age groups under 65 years and 65 years or over, ${ }^{14}$ local spread at the time of surgery, ${ }^{22}$ and survival pattern after surgery, ${ }^{22}$ we did additional post hoc exploratory analyses to investigate such effects. Because the outcomes of cancer are usually summarised in terms of five year survival and five year disease-free survival, we also examined these end points, initially with two by two tables assessed with the $\chi^{2}$ test and then in a multivariate logistic model with recurrence of cancer or death from any cause within five years of surgery as the end point. We express results as either hazard ratios or risk ratios with $95 \%$ confidence intervals.

Subgroup analyses assessed patients' sex, age groups, and each major organ surgery. For these factors, we tested for interaction by using the Breslow-Day test for homogeneity of odds ratios across strata. ${ }^{23}$ Because previous studies have suggested a variable effect of epidural block over shorter $(<1.5$ years) and longer time frames after surgery, ${ }^{22}$ we assessed this effect in our dataset. We used SPSS for Windows, version 18 for analyses.

\section{RESULTS}

Of the original 915 eligible patients in the MASTER trial, 163 had vascular surgery alone and 246 patients had major abdominal surgery for non-cancer causes; 
Table 1| Characteristics of patients at entry. Values are numbers (percentages) unless stated otherwise

\begin{tabular}{|c|c|c|}
\hline & $\begin{array}{l}\text { Epidural } \\
(n=230)\end{array}$ & $\begin{array}{c}\text { No epidural } \\
(\mathrm{n}=216)\end{array}$ \\
\hline \multicolumn{3}{|l|}{ Demographic characteristics } \\
\hline Mean (SD) age (years) & $71(9.5)$ & $70(11)$ \\
\hline \multicolumn{3}{|l|}{ Age group (years): } \\
\hline$\leq 50$ & $5(2)$ & $11(5)$ \\
\hline $51-60$ & $26(11)$ & $30(14)$ \\
\hline $61-70$ & $75(33)$ & $71(33)$ \\
\hline $71-80$ & $87(38)$ & $75(35)$ \\
\hline 180 & $37(16)$ & $29(13)$ \\
\hline Male sex & $138(60.0)$ & $114(53)$ \\
\hline \multicolumn{3}{|l|}{ Site of surgery } \\
\hline Oesophagogastric & $24(10)$ & $34(15)$ \\
\hline Hepatobiliary/pancreatic & $34(15)$ & $28(13)$ \\
\hline Bowel & $128(56)$ & $108(50)$ \\
\hline Renal & $11(5)$ & $19(9)$ \\
\hline Prostate/bladder & $8(3)$ & $3(1)$ \\
\hline Uterine/ovarian & $20(9)$ & $20(9)$ \\
\hline Other & $5(2)$ & $5(2)$ \\
\hline \multicolumn{3}{|l|}{ Characteristics of cancer } \\
\hline Large bowel: & $(n=58)$ & $(n=54)$ \\
\hline Duke's A & $12(21)$ & $12(22)$ \\
\hline Duke's B & $27(47)$ & $23(43)$ \\
\hline Duke's C & $19(33)$ & $23(43)$ \\
\hline TNM classification: & $(n=125)$ & $(n=106)$ \\
\hline $\mathrm{T} 1$ & $27(22)$ & $16(15)$ \\
\hline $\mathrm{T} 2$ & $25(20)$ & $30(28)$ \\
\hline $\mathrm{T} 3$ & $70(56)$ & $52(49)$ \\
\hline $\mathrm{T} 4$ & $3(2)$ & $8(8)$ \\
\hline NO & $81(65)$ & $68(64)$ \\
\hline N1 & $35(28)$ & $27(25)$ \\
\hline N2 & $10(8)$ & $11(10)$ \\
\hline Tumour grade: & $(n=145)$ & $(n=140)$ \\
\hline Well to moderately differentiated & $16(11)$ & $17(12)$ \\
\hline Poorly differentiated & $102(70)$ & $89(64)$ \\
\hline Undifferentiated & $27(19)$ & $34(24)$ \\
\hline \multicolumn{3}{|l|}{ Pre-existing medical conditions } \\
\hline Morbid obesity & $7(3)$ & $7(3)$ \\
\hline Chronic obstructive lung disease & $25(11)$ & $17(8)$ \\
\hline Previous myocardial infarction & $23(10)$ & $15(7)$ \\
\hline Diabetes & $114(50)$ & $122(56)$ \\
\hline Heart failure & $26(11)$ & $16(7)$ \\
\hline Hepatic failure & $23(10)$ & $19(9)$ \\
\hline Renal failure & $5(2)$ & $10(5)$ \\
\hline \multicolumn{3}{|l|}{ Blood tests-mean (SD) } \\
\hline Sodium (mmol/L) & $140(3.6)$ & $139(4.0)$ \\
\hline Potassium (mmol/L) & $4.3(0.5)$ & $4.2(0.5)$ \\
\hline Creatinine $(\mu \mathrm{mol} / \mathrm{L})$ & 109 (119) & $106(78)$ \\
\hline Urea (mmol/L) & $6.6(4.1)$ & $6.6(3.7)$ \\
\hline Albumin (mmol/L) & $36(5.8)$ & $35(6.0)$ \\
\hline Total protein $(\mathrm{g} / \mathrm{L})$ & $70(8.2)$ & $70(7.4)$ \\
\hline Bilirubin (mmol/L) & $36(82)$ & $37(91)$ \\
\hline Aspartate transaminase (mmol/L) & $32(30$ & $57(96)$ \\
\hline Alkaline phosphatase (mmol/L) & $169(223)$ & $169(258)$ \\
\hline Haemoglobin (mg/L) & $12.3(2.0)$ & $12.3(1.8)$ \\
\hline Platelets $\left(\times 10^{9} / \mathrm{L}\right)$ & $286(117)$ & $277(84)$ \\
\hline White cell count $\left(\times 10^{9} / \mathrm{L}\right)$ & $7.8(2.6)$ & $7.8(2.4)$ \\
\hline International normalised ratio & $1.0(0.1)$ & $1.0(0.1)$ \\
\hline Activated partial thromboplastin time (s) & $30(6.4)$ & $30(6.6)$ \\
\hline
\end{tabular}

31 had distant metastases at the time of surgery and thus had incompletely resectable cancer; a further three patients were unclassified (fig 1). Patients were followed up from March 2009 to May 2010. No patient or next of kin refused; one local medical practitioner refused to attempt to contact a patient who was thought to be alive, not having seen the patient for more than eight years. Some centres were unable to follow up patients because medical records had been destroyed and electronic hospital databases were unavailable; we were thus unable to determine survival status or date of death for a further 24 patients. Only four $(<1 \%)$ of the patients had incomplete (censored) data within the first five years after surgery.

Table 1 shows the distribution of perioperative risk factors in both groups. Baseline characteristics of the two groups, as might be expected from the randomised design, were comparable in each group. Types of cancer surgery, and measures of tumour invasiveness and differentiation, were also similar in the two groups.

Patients assigned to epidural anaesthesia were given more intravenous fluid and more red cell transfusions, as might be expected from the sympathetic block associated with effective epidural anaesthesia (table 2). The median maximal block height over the first three days after surgery in the epidural group was thoracic dermatome $\mathrm{T} 4$ (interquartile range $\mathrm{T} 2-\mathrm{T} 6$, range $\mathrm{T} 1-\mathrm{T} 10$ ). Four patients received at least one dose of pethidine, and three were each given a single dose of fentanyl. Median morphine sulphate equivalent usage over the first 72 hours after surgery was 0 (interquartile range 0 31) $\mathrm{mg}$ for epidural patients and 107 (44-202) $\mathrm{mg}$ for control patients $(\mathrm{P}<0.001)$.

The median time to recurrence of cancer or death was 2.6 (interquartile range $0.7-8.7$ ) years in the epidural group and $2.8(0.7-8.7)$ years in the non-epidural group $(\mathrm{P}=0.61)$. Recurrence-free survival was similar in both epidural and non-epidural groups (hazard ratio $0.95,95 \%$ confidence interval 0.76 to 1.17 ; $\mathrm{P}=0.61$ (fig 2). Five year recurrence and mortality rates were comparable (table 3 ). We found no interaction across each of the three pre-defined subgroups (fig 3, all $\mathrm{P}>0.10$ ). Recurrence-free survival remained essentially unchanged when restricted to 1.5 years after surgery (hazard ratio $0.95,0.75$ to $1.36 ; \mathrm{P}=0.95$ ). An analysis that included all the additional patients who had distant metastatic disease at the time of surgery but still had complete resection of the primary cancer $(\mathrm{n}=22)$ showed a similar risk of cancer-free survival (hazard ratio $0.94,0.76$ to $1.16 ; \mathrm{P}=0.56$ ).

Cox regression identified significant predictors of early death or recurrence of cancer as patient's age $(\mathrm{P}<0.001)$, female sex (hazard ratio $0.65,0.52$ to 0.82 ; $\mathrm{P}<0.001$ ), and red cell transfusion (hazard ratio 0.63 , 0.47 to $0.84 ; \mathrm{P}=0.002$ ) but not epidural group (hazard ratio $1.04,0.84$ to $1.30 ; \mathrm{P}=0.72)$. After adjustment for age and sex, type of cancer surgery had no significant effect on cancer-free survival $(\mathrm{P}=0.31)$. These estimates were similar after inclusion of the degree of differentiation of the cancer, tumour-nodes-metastases classification, or both at the time of surgery. Causes of death did 
Table 2 |Post-randomisation perioperative characteristics

\begin{tabular}{|c|c|c|c|}
\hline & Epidural $(n=230)$ & No epidural $(n=216)$ & $P$ value \\
\hline \multicolumn{4}{|l|}{ Intraoperative factors } \\
\hline Mean (SD) lowest temperature $\left({ }^{\circ} \mathrm{C}\right)$ & $35.5(0.8)$ & $35.6(0.8)$ & 0.16 \\
\hline Mean (SD) lowest blood pressure $(\mathrm{mm} \mathrm{Hg})$ & $87(15)$ & $93(17)$ & $<0.001$ \\
\hline Mean (SD) highest heart rate (beats/min) & $90(17)$ & $93(20)$ & 0.052 \\
\hline Median (IQR) crystalloid (mL) & $2500(2000-3725)$ & $2400(1500-3000)$ & 0.11 \\
\hline Median (IQR) colloid (mL) & $0(0-1000)$ & $0(0-500)$ & 0.005 \\
\hline No $(\%)$ red cell transfusion & $45(20)$ & $27(13)$ & 0.043 \\
\hline No (\%) blood product transfusion & $46(20)$ & $29(13)$ & 0.064 \\
\hline Median (IQR) blood loss (mL) & $300(90-600)$ & $300(100-600)$ & 0.39 \\
\hline Mean (SD) urine output (mL) & $510(456)$ & 369 (341) & 0.003 \\
\hline \multicolumn{4}{|l|}{ Postoperative pain scores_mean (SD) } \\
\hline \multicolumn{4}{|l|}{ Postoperative day 1 (am): } \\
\hline At rest & $1.8(2.4)$ & $2.5(2.3)$ & 0.006 \\
\hline With coughing & $4.0(3.3)$ & $5.5(2.6)$ & $<0.001$ \\
\hline \multicolumn{4}{|l|}{ Postoperative day 1 (pm): } \\
\hline At rest & $1.6(2.3)$ & $2.1(2.3)$ & 0.042 \\
\hline With coughing & $4.0(3.0)$ & $5.1(2.6)$ & $<0.001$ \\
\hline \multicolumn{4}{|l|}{ Postoperative day 2 (am): } \\
\hline At rest & $1.4(2.1)$ & $1.5(1.9)$ & 0.59 \\
\hline With coughing & $3.7(2.8)$ & $4.6(2.6)$ & 0.002 \\
\hline \multicolumn{4}{|l|}{ Postoperative day $2(\mathrm{pm}):$} \\
\hline At rest & $1.3(1.9)$ & $1.5(2.1)$ & 0.27 \\
\hline With coughing & $3.3(2.5)$ & $4.3(2.8)$ & 0.001 \\
\hline \multicolumn{4}{|l|}{ Postoperative day 3 (am): } \\
\hline At rest & $1.1(1.8)$ & $1.1(1.7)$ & 0.90 \\
\hline With coughing & $3.1(2.6)$ & $3.8(2.6)$ & 0028 \\
\hline \multicolumn{4}{|l|}{ Postoperative day $3(\mathrm{pm}):$} \\
\hline At rest & $1.0(1.7)$ & $1.1(1.9)$ & 0.62 \\
\hline With coughing & $3.3(2.6)$ & $3.6(2.7)$ & 0.063 \\
\hline
\end{tabular}

$\mathrm{IQR}=$ interquartile range for evaluating the independent effects of epidural block on various types of cancer, we also found no evidence that regional analgesia reduced recurrence of cancer or mortality for any subgroup included in our analysis.

The major putative mechanisms by which regional block might reduce the risk of recurrence of cancer are decreasing the surgical stress response, reducing the requirement for volatile anaesthesia, and obviating the need for opioid administration. Nearly all our patients were given thoracic epidural analgesia. The thoracic approach blocks the sympathetic nervous system and ameliorates immune suppression induced by surgical stress much better than does lumbar epidural anaesthesia ${ }^{25}$; it was thus ideal for our purpose. The MASTER trial did not compare epidural anaesthesia alone with general anaesthesia alone. Instead, the comparison was between combined epidural and general anaesthesia and general anaesthesia alone. The dose of volatile anaesthetic was not recorded in the MASTER trial but surely was reduced in the patients who received epidural anaesthesia. ${ }^{26}$ Finally, postoperative epidural analgesia was effective in that the median dose of opioid (in morphine sulphate equivalents) was $0 \mathrm{mg}$ in the epidural group compared with a median of $107 \mathrm{mg}$ in the patients who had general anaesthesia alone.

We identified other factors that were associated with poorer cancer-free survival after surgery. These included older age, female sex, tumour-nodesmetastases status (results not shown), and intraoperative red cell transfusion. These exploratory findings are consistent with many previous studies and support the internal validity of our dataset, as well as providing confidence that we have not missed a true independent effect of epidural block.

not differ significantly between groups $(\mathrm{P}=0.83)$; cancer was the primary cause in $62 \%$ of epidural patients and $57 \%$ of non-epidural patients (table 3 ).

\section{DISCUSSION}

Anaesthetics and cancer recurrence

Host defence is well established as the primary determinant of progression of cancer, and function of natural killer cells is the single most important component. The stress response to surgery, volatile anaesthesia, and opioid administration all directly impair natural killer cell function, ${ }^{36}$ and each effect is ameliorated by spinal/epidural anaesthesia. In vitro, animal, and some observational human data suggested that epidural block would substantially reduce the risk of recurrence of cancer. ${ }^{31-13}$ Our results, though, fail to support this hypothesis: recurrence-free survival and mortality were nearly identical in both groups.

Bowel cancer is the third most common non-skin cancer diagnosed in Australia and most other parts of the world. ${ }^{24}$ That most of our patients had colon cancer is thus unsurprising, especially as colectomies are highly suitable for epidural anaesthesia. Tumour biology varies considerably from organ to organ, and the effects of regional block, if any, are likely to vary from site to site. Although we had extremely limited power

\section{Comparison with other studies}

Several small observational studies have evaluated the effects of regional analgesia on recurrence of cancer; some reported benefit, ${ }^{12}{ }^{13}$ whereas others did not. ${ }^{14-16}$ However, no study randomly assigned patients to regional analgesia; each was thus affected by selection bias and confounding, which are inherent in observational analyses. ${ }^{27}$ Recurrence rates have only once been reported in patients who were randomised to

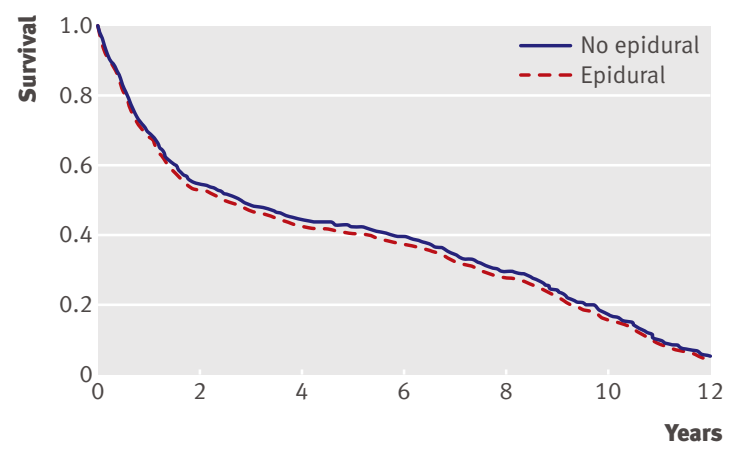

Fig $2 \mid$ Recurrence-free survival after cancer surgery by group (log rank $\mathrm{P}=0.61$ ) 


\begin{tabular}{|c|c|c|c|c|}
\hline & $\begin{array}{l}\text { Epidural } \\
(\mathrm{n}=230)\end{array}$ & $\begin{array}{l}\text { No epidural } \\
(n=216)\end{array}$ & $\begin{array}{l}\text { Hazard ratio or risk ratio } \\
\qquad(95 \% \mathrm{Cl})\end{array}$ & $P$ value \\
\hline \multicolumn{5}{|l|}{ Primary end point } \\
\hline Median $(95 \% \mathrm{Cl})$ recurrence-free survival (years) & $2.6(1.0$ to 4.7$)$ & $2.8(1.7$ to 3.8$)$ & $0.95(0.76$ to 1.17$)$ & 0.61 \\
\hline \multicolumn{5}{|l|}{ Secondary end points } \\
\hline Median $(95 \% \mathrm{Cl})$ time to recurrence (years) & $1.1(0.7$ to 1.6$)$ & $1.4(0.6$ to 2.3$)$ & $0.63(0.39$ to 1.02$)$ & 0.056 \\
\hline Median $(95 \% \mathrm{Cl})$ survival (years) & $3.3(2.1$ to 4.5$)$ & $3.7(2.0$ to 5.4$)$ & 0.95 (0.77 to 1.18$)$ & 0.66 \\
\hline \multicolumn{5}{|l|}{ At 5 years } \\
\hline Survival & $96(42)$ & $94(44)$ & $0.96(0.79$ to 1.17$)$ & 0.70 \\
\hline Recurrence-free survival & $92(40)$ & $92(43)$ & $0.95(0.78$ to 1.15$)$ & 0.58 \\
\hline \multicolumn{5}{|l|}{ Cause of death } \\
\hline Cancer & $114 / 183(62)$ & $97 / 169(57)$ & - & \multirow{5}{*}{0.83} \\
\hline Cardiovascular disease & $25 / 183(14)$ & $27 / 169(16)$ & - & \\
\hline Sepsis & $5 / 183(3)$ & 4/169 (2) & - & \\
\hline Other & 20/183 (11) & 19/169 (11) & - & \\
\hline Indeterminate or unknown & 19/183 (10) & $23 / 169(14)$ & - & \\
\hline
\end{tabular}

epidural analgesia or not. ${ }^{22}$ However, only 145 patients with non-metastatic disease were included and the results were equivocal, showing improved survival up to 1.5 years with no difference thereafter.

\section{Strengths and limitations of study}

A strength of this study is that we compared its 9-15 years' outcomes in a relatively large group of patients who were randomly assigned to general anaesthesia combined with epidural anaesthesia and analgesia versus general anaesthesia and opioid analgesia. Our sample size was limited by the size of the original MASTER trial and the fraction of those patients who had potentially curative surgery for cancer. Furthermore, follow-up data were not available for a small fraction of the patients for various reasons. Our analysis was thus restricted to 230 patients in the epidural

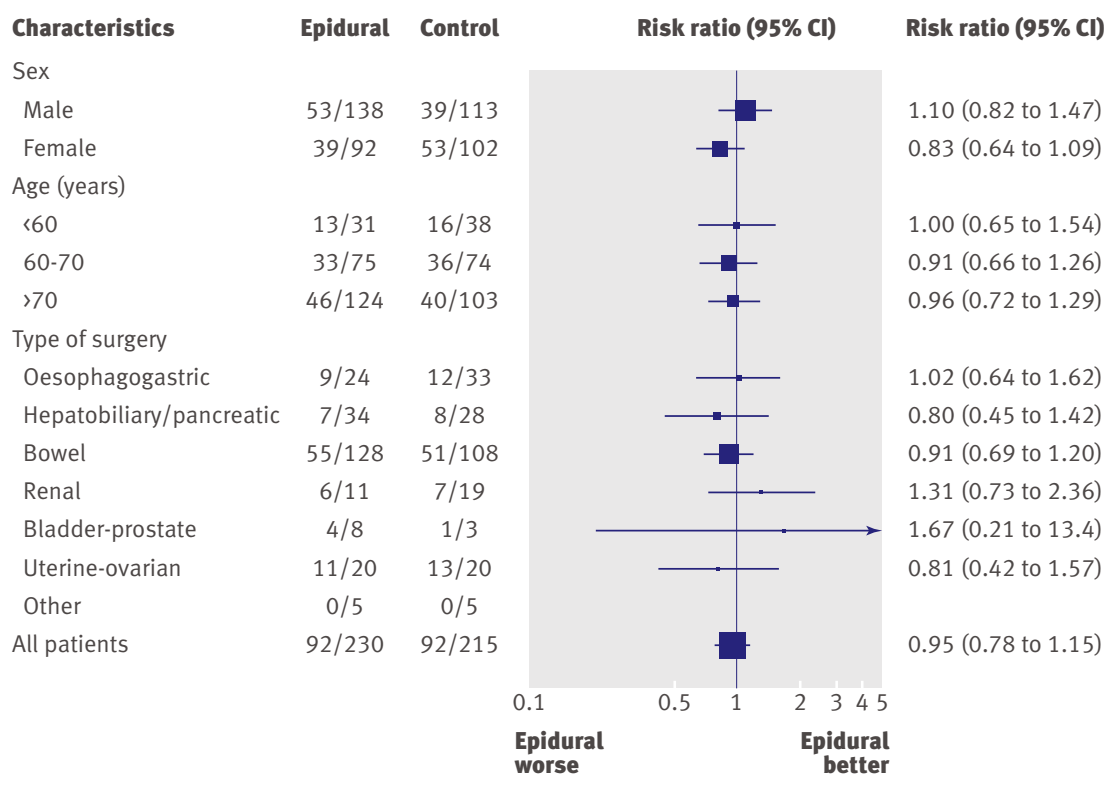

Fig 3 Five year recurrence-free survival by subgroups. Tests for interaction were not significant (all $P>0.10$ ) group and 215 patients in the non-epidural group. It was well powered to detect a one third treatment effect but lacked power to reliably detect smaller effects that might still be of considerable clinical importance, particularly for individual types of cancer. Our study does not provide information on the effects of regional blockade on non-abdominal (for example, breast) cancers. Additional large trials are thus clearly warranted. Several prospective randomised trials of regional analgesia and recurrence of cancer have started in recent years (NCT00684229, NCT00418457, and NCT01179308). However, given the time needed for enrolment of patients and prolonged observation for recurrence, results will probably not be available for five years or longer. Our results are likely to be the only randomised data on this subject for years to come. At the very least, our results suggest that in vitro, animal, and observational human data about regional block and recurrence of cancer should be extrapolated to patients with considerable caution. Although compelling short term reasons exist to use epidural analgesia in many patients, reducing the long term risk of recurrence of cancer may not be among them.

Concerns have been raised about the expertise and management of the epidural analgesia provided to patients in the original MASTER trial. ${ }^{28} \mathrm{We}$ thus provide some additional information in our methods section. The failure rate of insertion was very low (4.6\%), and in any case we could show effective epidural block, reduced pain scores, and a substantial reduction in opioid administration in the postoperative period.

Conclusions and clinical decision making implications In summary, we report the first long term follow-up of recurrence of cancer in patients assigned to general anaesthesia combined with epidural anaesthesia and analgesia or to general anaesthesia with opioid analgesia. Cancer recurrence rates and mortality were nearly identical in each group. The study had adequate power to detect relative risk reductions of about a third but 


\section{WHAT IS ALREADY KNOWN ON THIS TOPIC}

Recent observational studies have found a strong association between use of local anaesthetic (regional) block for cancer surgery and reductions in late recurrence of cancer

Whether selection of patients, other perioperative factors, or detection bias can explain these findings is unclear

\section{WHAT THIS STUDY ADDS}

This randomised trial could not identify any reduction in recurrence of cancer or survival when epidural block was used for surgery for abdominal cancer

lacked power for smaller effects that might still be clinically important. The decision to use epidural block in combination with general anaesthesia for major surgery for abdominal cancer should be based on other considerations. For example, epidural analgesia might be chosen to limit opioid related side effects or avoided to reduce the risk of hypotension and additional need for intravenous fluids.

Konrad Jamrozik (KJ) was a principal investigator for the MASTER trial and was instrumental in establishing and advising on this cancer recurrence study. Unfortunately, he passed away before the completion of the study. We thank Barbara Chan, Data Linkage Unit, Australian Institute of Health and Welfare, Canberra, Australia, for her assistance with some data collection and Richard Parsons for facilitating ethics approval and data management in Western Australia. Investigators and participating sites are listed as a web extra on bmj.com.

Contributors: JRAR and KJ conceived the original MASTER trial, and DIS conceived the follow-up cancer study. PSM, DIS, KJ, BS, and PP contributed to study design. JH coordinated the data collection. PSM did the statistical analyses. PSM and DIS wrote the first draft of the report. PSM, PP, BS, JRAR, and DIS participated in interpreting the results and editing the report. PSM is the guarantor.

Funding: The Australian National Health and Medical Research Council funded the MASTER trial. The cancer follow-up study was funded by the Australian and New Zealand College of Anaesthetists, the Alfred Hospital Research Trust (Melbourne, Australia), and the Department of Outcomes Research at the Cleveland Clinic (OH, USA). PSM is funded by an Australian National Health and Medical Research Council practitioner fellowship. The funders had no role in study design, data collection, data analysis, data interpretation, or writing of the report.

Competing interests: All authors have completed the Unified Competing Interest form at www.icmje.org/coi_disclosure.pdf (available on request from the corresponding author) and declare: no support from any organisation for the submitted work; no financial relationships with any organisations that might have an interest in the submitted work in the previous three years; no other relationships or activities that could appear to have influenced the submitted work.

Ethical approval: For this follow-up study, additional ethics committee approval was given by each of the original MASTER sites, the Australian Institute of Health and Welfare (which is responsible for the National Death Index), and cancer councils in each state of Australia. Data sharing: Technical appendix, statistical code, and dataset are available from the corresponding author at p.myles@alfred.org.au. Participants' consent was not obtained, but the presented data are anonymised and risk of identification is low.

1 Denis MG, Lipart C, Leborgne J, LeHur PA, Galmiche JP, Denis M, et al. Detection of disseminated tumor cells in peripheral blood of colorectal cancer patients. Int / Cancer 1997;74:540-4.

2 Shakhar G, Ben-Eliyahu S. Potential prophylactic measures against postoperative immunosuppression: could they reduce recurrence rates in oncological patients? Ann Surg Oncol 2003;10:972-92.

3 Bar-Yosef S, Melamed R, Page GG, Shakhar G, Shakhar K, Ben-Eliyahu S. Attenuation of the tumor-promoting effect of surgery by spinal blockade in rats. Anesthesiology 2001;94:1066-73.

4 Zetter BR. Angiogenesis and tumor metastasis. Annu Rev Med 1998;49:407-24.
5 Antoni MH, Lutgendorf SK, Cole SW, Dhabhar FS, Sephton SE, McDonald PG, et al. The influence of bio-behavioural factors on tumour biology: pathways and mechanisms. Nat Rev Cancer 2006;6:240-8.

6 Sacerdote P, Bianchi M, Gaspani L, Manfredi B, Maucione A, Terno G, et al. The effects of tramadol and morphine on immune responses and pain after surgery in cancer patients. Anesth Analg 2000;90:1411-4.

7 Gupta K, Kshirsagar S, Chang L, Schwartz R, Law PY, Yee D, et al. Morphine stimulates angiogenesis by activating proangiogenic and survival-promoting signaling and promotes breast tumor growth. Cancer Res 2002;62:4491-8.

8 Ben-Eliyahu S, Page GG, Yirmiya R, Shakhar G. Evidence that stress and surgical interventions promote tumor development by suppressing natural killer cell activity. Int / Cancer 1999;80:880-8.

9 O'Riain SC, Buggy DJ, Kerin MJ, Watson RW, Moriarty DC. Inhibition of the stress response to breast cancer surgery by regional anesthesia and analgesia does not affect vascular endothelial growth factor and prostaglandin E2. Anesth Analg 2005;100:244-9.

10 Chae BK, Lee HW, Sun K, Choi YH, Kim HM. The effect of combined epidural and light general anesthesia on stress hormones in open heart surgery patients. Surg Today 1998;28:727-31.

11 Page GG, Blakely WP, Ben-Eliyahu S. Evidence that postoperative pain is a mediator of the tumor-promoting effects of surgery in rats. Pain 2001:90:191-9.

12 Exadaktylos AK, Buggy DJ, Moriarty DC, Mascha E, Sessler DI. Can anesthetic technique for primary breast cancer surgery affect recurrence or metastasis? Anesthesiology 2006;4:660-4.

13 Biki B, Mascha E, Moriarty DC, Fitzpatrick JM, Sessler DI, Buggy DJ. Anesthetic technique for radical prostatectomy surgery affects cancer recurrence: a retrospective analysis. Anesthesiology 2008;109:180-7.

14 Gottschalk A, Ford JG, Regelin CC, You J, Mascha EJ, Sessler DI, et al. Association between epidural analgesia and cancer recurrence after colorectal cancer surgery. Anesthesiology 2010;113:27-34.

15 Tsui BC, Rashiq S, Schopflocher D, Murtha A, Broemling S, Pillay J, et al. Epidural anesthesia and cancer recurrence rates after radical prostatectomy. Can J Anaesth 2010;57:107-12.

16 Ismail H, Ho KM, Narayan K, Kondalsamy-Chennakesavan S. Effect of neuraxial anaesthesia on tumour progression in cervical cancer patients treated with brachytherapy: a retrospective cohort study. $\mathrm{Br}$ I Anaesth 2010;105:145-9.

17 Wuethrich PY, Hsu Schmitz SF, Kessler TM, Thalmann GN, Studer UE, Stueber $F$, et al. Potential influence of the anesthetic technique used during open radical prostatectomy on prostate cancer-related outcome: a retrospective study. Anesthesiology 2010;113:570-6.

18 Rigg JRA, Jamrozik K, Myles PS, Silbert B, Peyton P, Parsons RW, et al. Design of the multicenter Australian study of epidural anesthesia and analgesia in major surgery: the MASTER Trial. Control Clin Trials 2000;21:244-56.

19 Rigg JRA, Jamrozik K, Myles PS, Silbert B, Peyton P, Parsons RW, et al. Epidural anaesthesia and analgesia and outcome of major surgery: a randomised trial. Lancet 2002;359:1276-82.

20 Peyton PI, Myles PS, Silbert BS, Rigg JRA, Jamrozik K, Parsons RW, et al. Perioperative epidural analgesia and outcome after major abdominal surgery in high risk patients. Anesth Analg 2003;96:548-54.

21 American Joint Committee on Cancer. AJCC cancer staging manual. 6th ed. Springer, 2002.

22 Christopherson R, James KE, Tableman M, Marshall P, Johnson FE. Long-term survival after colon cancer surgery: a variation associated with choice of anesthesia. Anesth Analg 2008;107:325-32.

23 Breslow NE, Day NE. Statistical methods in cancer research. Volume I: the analysis of case-control studies. IARC Sci Publ 1980;32:5-338.

24 Australian Institute of Health and Welfare. Cancer in Australia: an overview 2010. AlHW, 2010 (available at www.aihw.gov.au/ publications/can/56/12138.pdf). (Cancer series no 60.)

25 Ahlers O, Nachtigall I, Lenze J, Goldmann A, Schulte E, Höhne C, et al. Intraoperative thoracic epidural anaesthesia attenuates stressinduced immunosuppression in patients undergoing major abdominal surgery. Br / Anaesth 2008;101:781-7.

26 Hodgson PS, Liu SS. Epidural lidocaine decreases sevoflurane requirement for adequate depth of anesthesia as measured by the bispectral index monitor. Anesthesiology 2001;94:799-803.

27 Collins R, MacMahon S. Reliable assessment of the effects of treatment on mortality and major morbidity, Il: observational studies. Lancet 2001;357:455-62.

28 De Leon-Casasola OA. When it comes to outcome, we need to define what a perioperative epidural technique is. Anesth Analg 2003;96:315-8.

Accepted: 31 January 2011 